\title{
Hemorroidectomia Híbrida. Uma Nova Abordagem no Tratamento das Hemorróidas Mistas
}

\author{
Hybrid Hemorrhoidectomy - A New Approach in the \\ Treatment of Hemorrhoids
}

\author{
HAROLDOALFREDOSANTOS ${ }^{1}$ \\ ${ }^{1}$ Membro Associado da Sociedade Brasileira de Coloproctologia
}

SANTOS HA. Hemorroidectomia Híbrida. Uma Nova Abordagem no Tratamento das Hemorróidas Mistas. Rev bras Coloproct, 2006;26(4): 377-388.

RESUMO: Este trabalho tem por objetivo apresentar uma nova abordagem mini-invasiva das Hemorróidas Mistas, a Hemorroidectomia Híbrida, que consiste na associação da Ligadura Elástica (LE) das Hemorróidas Internas com a ressecção complementar dos Plicomas Externos sob anestesia local. Num universo de 326 cirurgias orificiais realizadas na Proctoclínica num período de 4 anos, $300(92 \%)$ foram submetidos a procedimentos mini-invasivos, $223(68,40 \%)$ foram submetidos a $\mathrm{LE}$ como tratamento exclusivo e $77(\mathbf{2 3 , 6 0 \%})$ à Hemoirroidectomia Híbrida) e $26(8 \%)$ foram submetidos a outros procedimentos cirúrgicos (Hemorroidectomias a Milligan-Morgan, Fistulectomias etc. A abordagem proposta permite absenteísmo mínimo ao trabalho, mini-invasividade e baixa morbidade pós-operatória, ressaltando-se ainda a realização ambulatorial, excelente tolerabilidade e baixos custos.

Descritores: Doença Hemorroidária; Hemorróidas; Hemorroidectomia; Ligadura Elástica.

\section{INTRODUÇÃO}

$\mathrm{O}$ alto custo e a carência de leitos hospitalares, associados a invasividade, absenteismo ao trabalho e a resistência dos pacientes à Hemorroidectomia clássica, vêm sugerindo, ao longo dos anos, a necessidade de se buscar novas opções para o tratamento da Doença Hemorroidária.

Em relação às Hemorróidas Internas, a Ligadura Elástica (LE) já preenche estes anseios, com menor invasividade, excelente tolerabilidade e eficácia comprovada, e hoje já há um consenso de que a LE é o tratamento de escolha para as Hemorróidas Internas, e a sua identificação com a etiopatogenia da Doença Hemorroidária (DH) é tão intrínseca que nos permite um aforisma de reforço: "A LE é, na verdade, uma Cirurgia Fisiológica".

Entretanto, em relação às Hemorrróidas Mistas, a Hemorroidectomia Clássica tem sido ainda a opção mais indicada.
Temos questionado se realmente as Hemorroidectomias Clássicas não estariam por merecer uma reavaliação à luz dos conceitos e tendências atuais de terapêuticas cada vez menos invasivas ${ }^{(66)}$, visto que as complicações muitas vezes podem ser piores que a própria $\mathrm{DH}$, e a necessidade de se adequar o tratamento à doença que vamos tratar, nos leva a reflexões importantes: Não estariam as Hemorroidectomias superdimensionadas em relação à patologia que vão tratar?

A cirurgia ideal é aquela que mais se identifica com a etiopatogenia da doença que vai tratar e cuja invasividade e morbidade sejam correspondentes à gravidade da referida doença.

Thomson" ${ }^{(59)}$, em seu trabalho "The Nature of Haemorrhoids", nos permite concluir que a "DH" é muito mais uma alteração anatômica e fisiopatológica do que uma "doença" propriamente dita, caracterizando-se pelo aumento de volume e perda de elasticidade das estruturas dos coxins hemorroidários, resultando

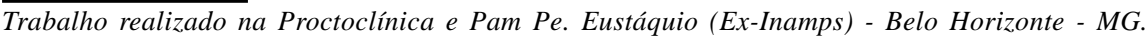

$\overline{\text { Recebido em 26/06/2006 }}$

Aceito para publicação em 14/07/2006 
em turgescência, dilatação vascular e deslizamento, e o que se busca no seu tratamento é a diminuição destes tecidos exuberantes e a fixação dos tecidos adjacentes, seja pela sua destruição por meios químicos (Escleroterapia) ou físicos (IRC), ou pela sua ressecção (Ligadura Elástica ou Hemorroidectomias Clássicas), e aqui aproveitamos para fixar um conceito que nos parece totalmente inequívoco: a LE é, na verdade, um Procedimento Cirúrgico, visto que é amputativo, com a "ressecção retardada" do mamilo hemorroidário.

Não incluímos o PPH nesta classificação porque esta técnica não resseca, nem destrói os mamilos hemorroidários e somente propõe a ressecção de uma faixa circular de mucosa e submucosa retais, $4 \mathrm{~cm}$ acima da linha pectínea e, portanto, acima dos coxins hemorroidários que seriam apenas suspensos na sua localização.

Ressaltamos o fato de que as Hemorróidas Internas são as principais responsáveis pela sintomatologia das Hemorróidas e os Plicomas Hemorroidários Externos são, quase sempre, secundários e dependentes das Hemorróidas Internas, e se tornam inertes após o tratamento das mesmas, daí a eficácia da LE no tratamento das Hemorróidas.

A LE permite a ressecção das Hemorróidas Internas sem a necessidade de feridas de "abordagem" e de "drenagem" através do canal anal (a abordagem convencional estipula estes princípios básicos na cirurgia orificial), e esta foi a grande evolução no sentido de se tratar as Hemorróidas com uma abordagem menos traumática e totalmente eficaz: a ligadura do conteúdo dos coxins hemorroidários com sua ressecção retardada, e a posterior cicatrização e fixação dos tecidos adjacentes, portanto, com a preservação do assoalho do canal anal.

Além de pouco invasiva a LE é também eclética na abordagem dos mamilos, e a sua realização em sessões múltiplas permite ressecar os mamilos escalonadamente e fixar a mucosa juxta-anal, reconstituindo a anatomia da região ano-retal.

Desde a década de 60, Parks $^{(16)}$ já propunha uma técnica de Hemorroidectomia Sub-mucosa em que os tecidos eram ressecados cirurgicamente por dissecção submucosa como alternativa de se preservar, ainda que parcialmente, a integridade do assoalho do canal anal, uma aspiração permanente da Coloproctologia. Entretanto, tecnicamente difícil, o procedimento exigia uma dissecção lenta e habilidosa, o que dificultou, senão impossibilitou, o seu uso de rotina.
Entretanto, a preservação da integridade do assoalho do canal anal continua como uma aspiração vigente e mais do que nunca nos compete, a nós proctologistas, utilizarmos técnicas mini-invasivas, cujas vantagens seriam:

a) Evitar as fissuras residuais e as estenoses iatrogênicas.

b) Diminuir a morbidade e as dores pós-operatórias.

c) Diminuir interferências ao ato defecatório e suas conseqüências.

d) Diminuir o absenteísmo ao trabalho.

E à simplificação dos procedimentos cirúrgicos acrescentaria outras vantagens:

e) Realização ambulatorial.

f) Diminuição dos custos.

\section{UMA NOVA ABORDAGEM NO TRATAMENTO DAS HEMORRÓIDAS MISTAS}

Respaldado no raciocínio lógico da miniinvasividade, idealizamos, a partir de 1986, uma abordagem cirúrgica múltipla das Hemorróidas Mistas que nos permitisse minimizar o trauma cirúrgico, procurando atingir os mesmos objetivos da Hemorroidectomia Clássica: a ressecção das Hemorróidas Internas e Externas, com a grande vantagem, na imensa maioria dos casos, da preservação do assoalho do canal anal pela ressecção complementar sub-cutânea do conteúdo dos Plicomas Externos após a LE das Hemorróidas Internas.

A este procedimento que associa técnicas cirúrgicas diferentes (LE nas Hemorróidas Internas e Exérese Complementar dos Plicomas Externos), convencionamos denominar "Hemorroidectomia Híbrida" pela analogia com o termo "Palavra Híbrida" usado na Língua Portuguesa para definir as palavras de origem múltipla.

Recentemente, Longo ${ }^{(67)}$ propôs uma nova abordagem para o tratamento das Hemorróidas Internas através do grampeador (PPH) com o mesmo objetivo de preservar o assoalho do canal anal, e que, da mesma forma que a LE, não trata os Plicomas Externos e, portanto, teria indicações semelhantes à LE. Entretanto enfatizamos que a LE é mais eclética por 
tratar cada mamilo individualmente, o que atenderia perfeitamente aos padrões de assimetria freqüentes na $\mathrm{DH}$, enquanto que o PPH teria uma limitação nas indicações, em virtude de seu padrão circular de ressecção, de certa forma pouco compatível com essa assimetria que estatisticamente constitue a maioria dos casos de Hemorróidas de III e IV graus.

Reforçando esta premissa, podemos citar o trabalho de Hayssen, T.K. and cols..$^{20}$ que propuseram a indicação da ressecção de 1 ou 2 mamilos mais sintomáticos como tratamento das Hemorróidas (115 pacientes) e realizaram um estudo prospectivo e randomizado comparando os resultados desta abordagem limitada com um grupo controle em que realizaram a Hemorroidectomia convencional dos 3 mamilos (133 pacientes), e num período de 8 anos de acompanhamento concluiram que não houve diferenças nos resultados dos 2 grupos, nem quanto ao desaparecimento dos sintomas após as cirurgias, nem quanto ao reaparecimento de sintomas a longo prazo.

Já abordamos este tema no $51^{\circ}$ Congresso Brasileiro de Coloproctologia (2002) ao participarmos da Mesa Redonda sobre Hemorróidas com o trabalho: "PPH x LE - Uma comparação inevitável".

O PPH propõe também, em casos indicados, a ressecção complementar dos Plicomas Externos, fato que somente reforça nossa proposta de abordagem múltipla e oligo-invasiva das Hemorróidas Mistas pela Hemorroidectomia Híbrida.

Reconhecemos que mudar um conceito arraigado ou um hábito que se transformou em rotina é bastante difícil; entretanto, o que nos parece mais difícil é mudar o "preconceito" dos Cirurgiões Coloproctologistas, acostumados ao longo de décadas a interpretar a Doença Hemorroidária como um "todo", quando, na verdade, ela é polimorfa e assimétrica, geralmente com um ou mais mamilos maiores e mais sintomáticos que os outros ${ }^{(20)}$, fato que só corrobora para a abordagem escalonada aos mamilos maiores e mais sintomáticos inicialmente, o que resulta numa melhora imediata que facilita a continuidade do tratamento em consultório, certamente a forma mais cômoda e preferida pelos pacientes.

Não podemos continuar alimentando o preconceito de que a cura da DH somente se daria com a ressecção radical e ampla das Hemorróidas através da Hemorroidectomia Clássica, porque percentualmente a cirurgia radical gera um número muito maior de complicações, e um dos preceitos da Medicina é exatamente evitar a iatrogenia.
É certo que em alguns casos de DH mais exuberante teríamos que indicar a Hemorroidectomia Clássica, mas nestes casos haveria uma correspondência entre a invasividade e morbidade cirúrgicas com a maior complexidade daquela Doença Hemorroidária em particular.

É importante reavaliar também um conceito muito difundido de que a LE teria suas indicações apenas para as Hemorróidas de II Grau, pois esta seria, a nosso ver, uma visão limitada da amplitude e da diversificação de suas indicações, subutilizando essa técnica versátil que, inclusive encontra outras indicações na própria Coloproctologia (Prolapso Retal, Papilites Hipertróficas, Pólipo Retal), bem como em outras especialidades médicas (Ligadura de Pedículo Umbelical, Ligadura de Trompa e Ligadura de Varizes Esofageanas Hemorrágicas).

Apesar da resistência quase inercial na ampliação das indicações, voltamos a insistir que nos compete criar as condições ideais para a realização da LE, e nós preconizamos realizá-la com o paciente na posição genu-peitoral, na qual teríamos a facilidade da re-introdução dos mamilos exteriorizados e o esvaziamento postural do seu conteúdo sanguíneo, conseguindo assim reduzir significantemente o volume de mamilos, o que facilita a sua apreensão pela campânula da Pinça de Ligadura, e talvez esta seja a diferença de conduta e o porque de alguns proctologistas utilizarem a LE apenas para os casos de Hemorróidas de II grau.

Corman (11), no seu trabalho "The New Hemorrhoidectomy", já afirmava que, em virtude da invasividade das Hemorroidectomias Clássicas e após três anos consecutivos de avaliação da tolerabilidade e resultados da Ligadura Elástica (1969 a 1972), a Lahey Clinic Foundation havia praticamente afastado indicações mais invasivas no tratamento das Hemorróidas, e somente 1 em cada 5 casos de "Hemorróidas Significativamente Grandes" eram submetidos à Hemorroidectomia, e complementava com uma observação de que talvez a invasividade e a dor como ocorrência pos-operatória mais frequiente, fosse a razão pela qual tão poucos Cirurgiões se submetiam à Hemorroidectomia Clássica, e também aqui entre nós não é diferente.

\section{MATERIAL E MÉTODOS}

Tendo exercido a Cirurgia Geral no ExINAMPS durante os primeiros 15 anos de atividade 
profissional, tivemos a oportunidade de realizar centenas de Cirurgias Ambulatoriais sob anestesia local (20 a 30 cirurgias/mês), e esta experiência nos facultou a oportunidade de implantar na Clínica Proctológica um protocolo para o tratamento cirúrgico ambulatorial das Hemorróidas Mistas, ampliando também as indicações aos casos em que havia outras patologias associadas como fissuras e papilites, principalmente escudados na resistência individual dos pacientes à internação hospitalar e à cirurgia convencional, bem como nas restrições clinico-cardiológicas à intervenção sob bloqueio, o que nos autorizava intervenções menos invasivas ainda que alternativas naquela época.

A ampliação das indicações foi progressiva e a divulgação dos resultados pelos próprios pacientes nos trazia novos pacientes, ávidos de solução para os seus sintomas, e que resistiam às indicações de cirurgias mais invasivas.

Uma certa complacência, tanto na indicação médica quanto na aceitação pelos próprios pacientes, nos estimulou a ampliar as referidas indicações e, para nossa surpresa, os resultados foram favoráveis com uma unanimidade na aprovação por parte dos pacientes.

É importante que se diga que não havia o sentido de cobaias nos referidos pacientes, mas, e tão somente, a intenção de se resolver os seus sintomas e melhorar sua qualidade de vida. e os resultados foram sempre melhores que a expectativa, e, apesar da multiplicidade de intervenções, a recuperação após cada mini-intervenção era rápida, com baixo índice de absenteísmo ao trabalho.

Ressalte-se o fato de que no pós-operatório das Hemorroidectomias Clássicas, muitas vezes, é necessário reintervir em Fissuras e Plicomas Residuais, e este fato funciona como um aval e somente reforça as indicações destas mini-intervenções múltiplas na Hemorroidectomia Híbrida.

É importante mencionar também que nos pacientes portadores de Hemorróidas Mistas com plicomas exuberantes que são refratários por opção pessoal ou por contra-indicação clínica a se submeterem a Hemorroidectomia Clássica, a LE das Hemorróidas Internas já contempla resultados bastante satisfatórios pela acentuada diminuição do volume dos Plicomas Externos, que se tornam assintomáticos e inertes ${ }^{(53)}$.

Saliente-se também que, após a LE das Hemorróidas Internas, as Ressecções Complementa- res dos Plicomas Hemorroidários Externos se tornam econômicas, diminuindo acentuadamente a área cruenta das feridas cirúrgicas, e permitindo, na maioria das vezes, a abordagem cirúrgica subcutânea dos componentes externos, com a preservação da integridade do assoalho do canal anal, diminuindo dessa forma a invasividade e melhorando a tolerabilidade, e adequando, portanto, o tratamento à verdadeira dimensão da Doença Hemorroidária.(Figuras 1 e 2)

\section{ESTRATÉGIA CIRÚRGICA}

No nosso protocolo a ressecção dos plicomas pode ser concomitante ou posterior à ligadura do conteúdo interno e, nos casos de patologias associadas, como fissuras ou papilites polipóides, há, muitas vezes, necessidade de cirurgia prévia com a posterior complementação pela LE nas Hemorróidas Internas.

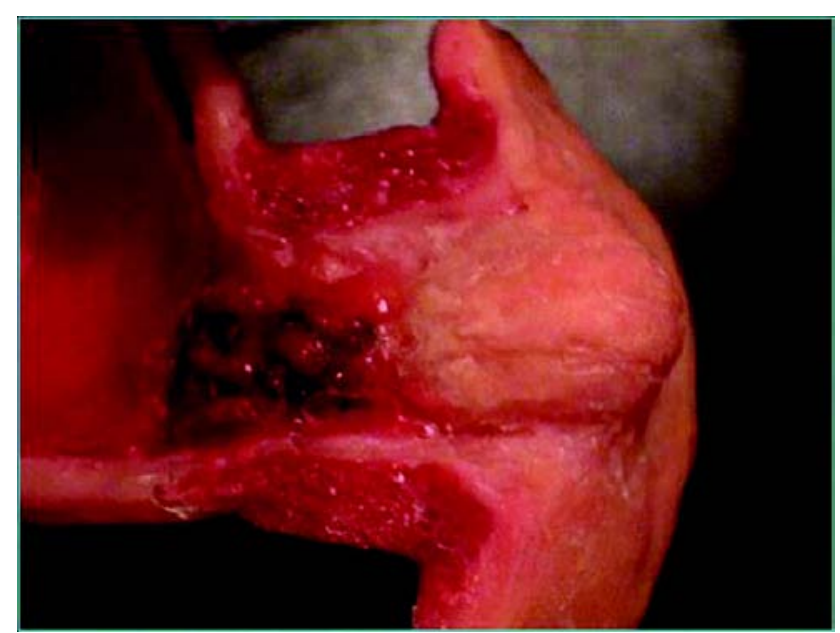

Figura 1 - Molde de Hemorróidas Mistas.

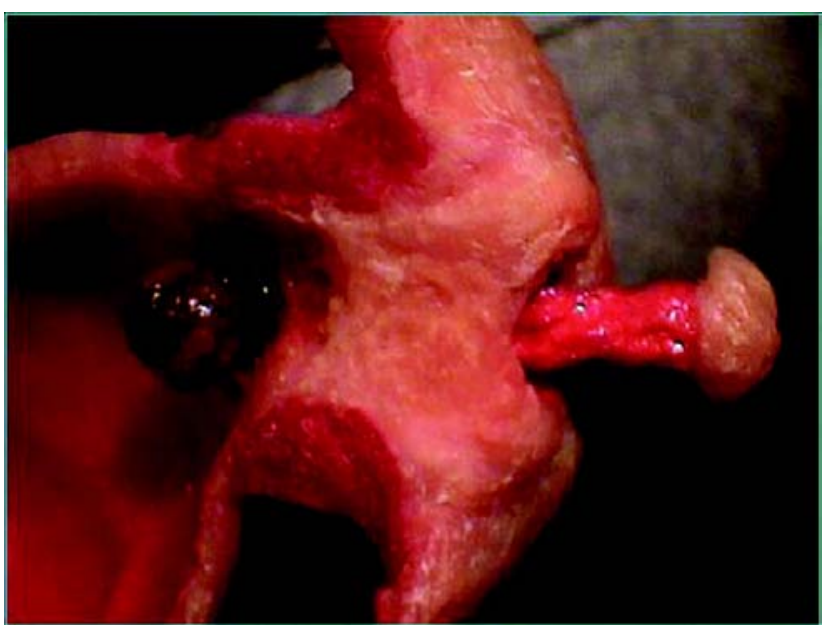

Figura 2-Molde de Exérese sub-cutânea do plicoma externo. 
Ressaltamos novamente a importância da posição genu-peitoral que permite o esvaziamento do conteúdo sanguíneo com a diminuição acentuada do volume dos coxins hemorroidários, ampliando a indicação da LE aos mamilos mais volumosos de III e IV graus.

Para a ressecção complementar dos componentes externos, utilizamos sempre a posição lateral com hiperflexão das coxas sobre o abdome com o objetivo de facilitar a abordagem à região ano-retal e permitir maior comodidade ao paciente, o que facilita a sua cooperação durante o ato cirúrgico, e, para a exposição, idealizamos uma perneira especial que denominamos "Perneira Horizontal", que possibilita a ampla exposição do períneo pelo afastamento das nádegas por meio de esparadrapo fixado em um arco auxiliar que faz conjunto com as perneiras

Esta perneira alcochoada amplia a extensão lateral da mesa cirúrgica e permite que as pernas nela se apóiem, permitindo maior comodidade, além da diminuição da pressão sobre os vasos da região ano-retal, diminuindo a congestão e facilitando a realização do ato cirúrgico. (Figura 3 )

\section{ANESTESIA TÓPICA NAS LIGADURAS ELÁSTICAS}

Para a realização da LE temos utilizado sistematicamente a anestesia tópica da região ano-retal, com a utilização de uma "mecha" de algodão embebida em anestésico tópico (Lidocaina Spray a 10\%) que é colocada ao nível da Linha Pectínea.

A absorção do anestésico é rápida e o efeito é quase imediato, diminuindo a sensibilidade local e facilitando a aplicação das ligaduras, e, dessa forma, abolimos os espasmos reflexos ano-sigmoideanos, bem como o Reflexo Vagal, muitas vezes responsável por bradicardias incômodas após o manuseio das estruturas ano-esfinterianas.

\section{ANESTESIA TÓPICA NAS CIRURGIAS COMPLEMENTARES}

A Exérese dos Plicomas Externos é realizada sob anestesia local, privilegiando o uso da menor quantidade possível de anestésico para evitar as deformações da anatomia anal e facilitar a abordagem cirúrgica dos referidos plicomas, com ressecções mais econômicas e feridas cirúrgicas menores que tornam a cirurgia menos invasiva.

Utilizamos há vários anos as seringas e agulhas de uso odontológico pelas características do bisel afilado com infiltrações menos traumáticas e anestésico em tubetes descartáveis de $2 \mathrm{ml}$.

É bom frisar que uma tática anestésica adequada, com menor volume de anestésico, possibilita ressecções mais estanques e feridas cirúrgicas menores com todo o séquito de vantagens: menor morbidade e recuperação mais rápida.

Nos casos de plicomas muito exuberantes cuja dissecção cirúrgica se prolonga ao canal anal, a área de ressecção é menor em virtude do "emagrecimento" dos plicomas devido à LE prévia do componente interno, e muitas vezes, após o tratamento das Hemorróidas Internas pela LE, a diminuição do volume dos Plicomas Externos é tão acentuada que acaba por afastar a necessidade de sua ressecção complementar, visto que há o desaparecimento completo dos sintomas e o plicoma se torna inerte. Figura 4.

Nos casos de Hemorróidas Internas de III e IV graus nos quais permanece apenas um deslizamento do assoalho do canal anal após o tratamento dos mamilos internos pela LE, o tratamento cirúrgico complementear é realizado pela simples dissecção subcutânea por divulsão que irá facultar uma posterior fixação cicatricial, reconstituindo o assoalho do canal anal. Figura 5.

O planejamento em mini-procedimentos múltiplos pode nos levar à falsa impressão de uma evolução

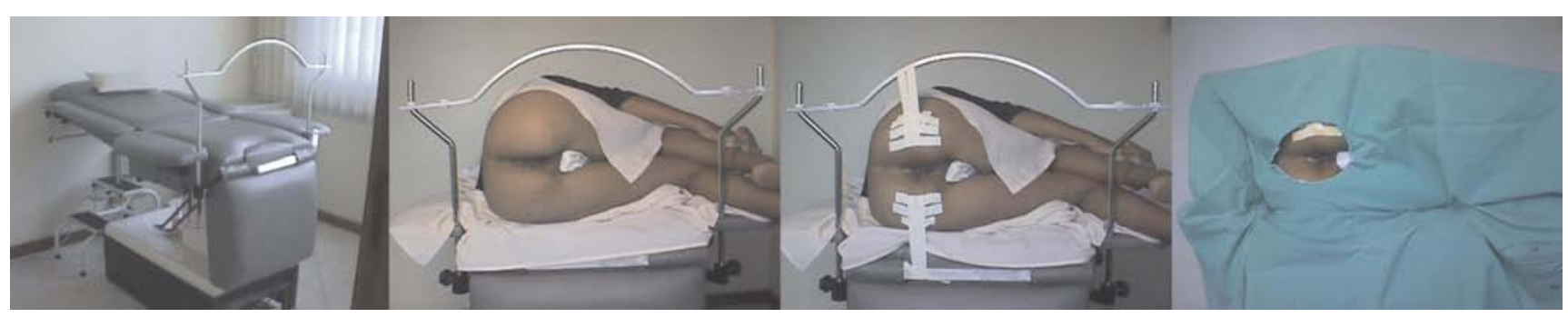

Figura 3 - Perneira Lateral e Posição Lateral com fixação das nádegas em arco auxiliar. 


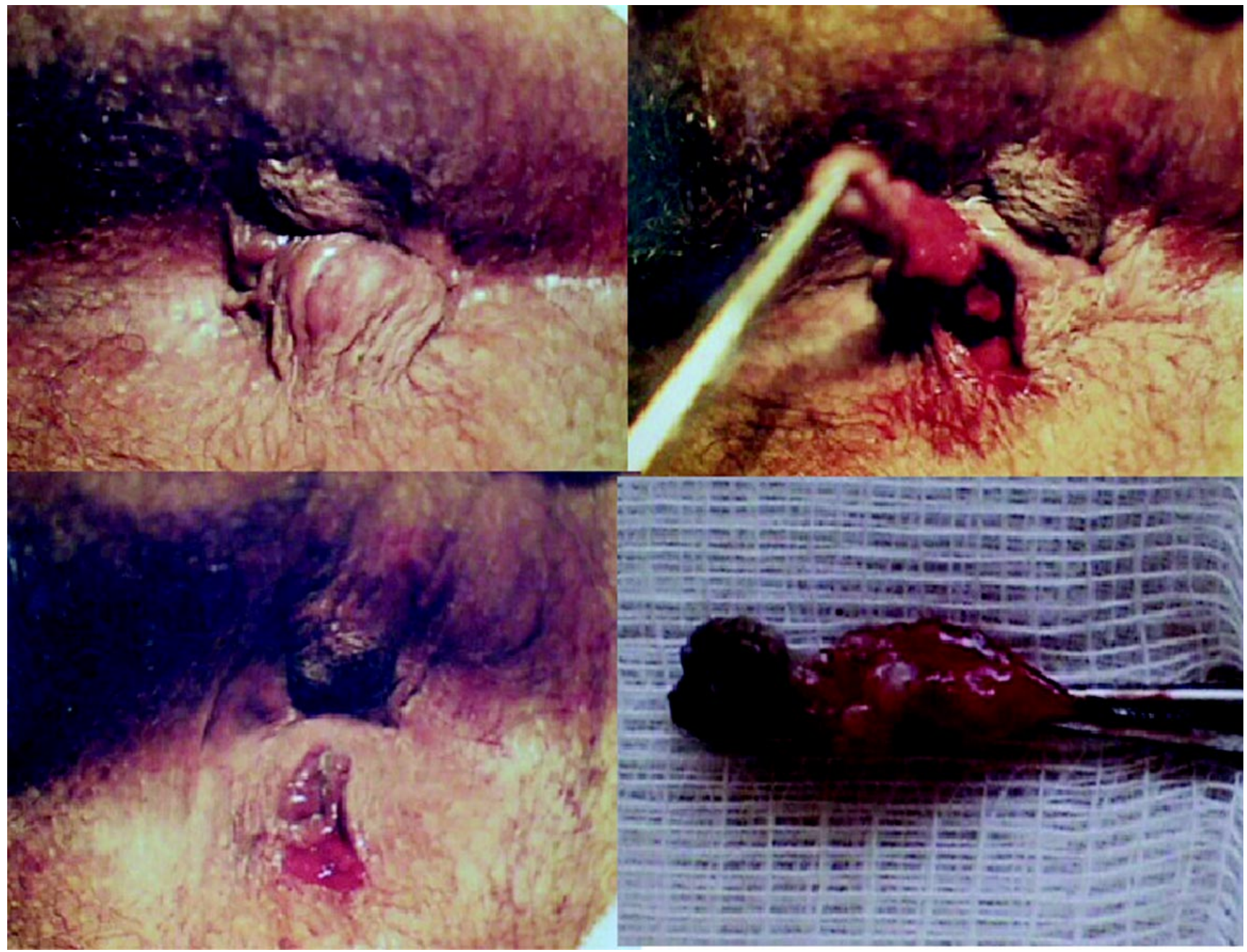

Figura 4 - Ressecção de Plicoma Externo com preservação do assoalho do canal anal.
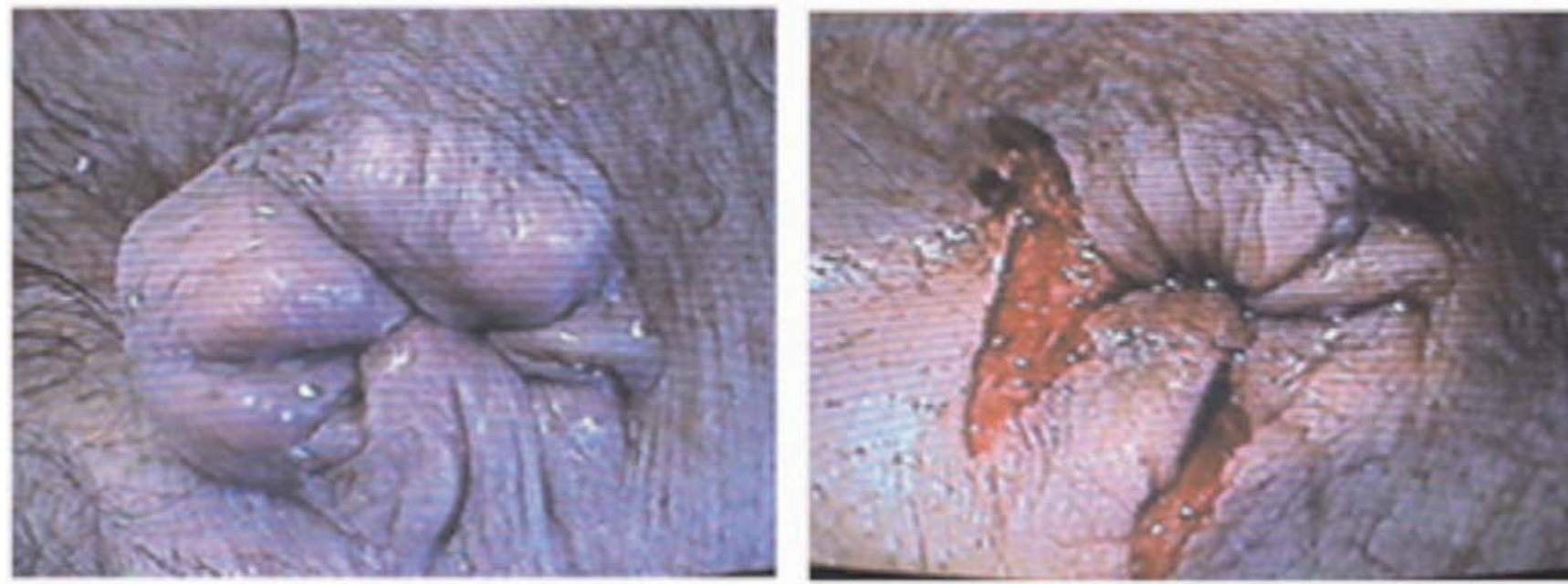

Figura 5 - Ressecção Complementar e dissecção subcutânea para fixação de assoalho anal deslizante após LE. 
complexa e prolongada. Entretanto, o que se verifica é o contrário, pequenos traumas com recuperação mais rápida e interstícios que permitem ao paciente retomar suas atividades normais entre as intervenções, diminuindo bastante o absenteísmo no trabalho, diferente do que acontece no p.o. da Hemorroidectomia Clássica, com seus curativos geralmente incômodos e limitantes das atividades pessoais e absenteísmo obrigatório nas atividades pessoais por períodos mais prolongados. Figuras 6,7 e 8 .

\section{CASUÍSTICA}

Realizamos a Hemorroidectomia Híbrida desde 1986, entretanto apresentamos neste trabalho um estudo retrospectivo referente aos pacientes operados na Proctoclínica no período de 1996 a 1999 e temos plena consciência da necessidade de se agregar a este levantamento retrospectivo um novo estudo prospectivo e, se possível, multi-cêntrico, com um protocolo bem elaborado que permita conclusões mais amplas e abrangentes. (Tabelas 1 a 6 )

\section{CONCLUSÃO}

Podemos concluir que não se trata de uma nova técnica cirúrgica, mas do dimensionamento, racionalização e associação de técnicas já disponíveis no arsenal terapêutico cirúrgico.

As nossas expectativas, firmadas no uso destas indicações e respaldadas na observação da evolução e resultados, têm sido satisfatoriamente

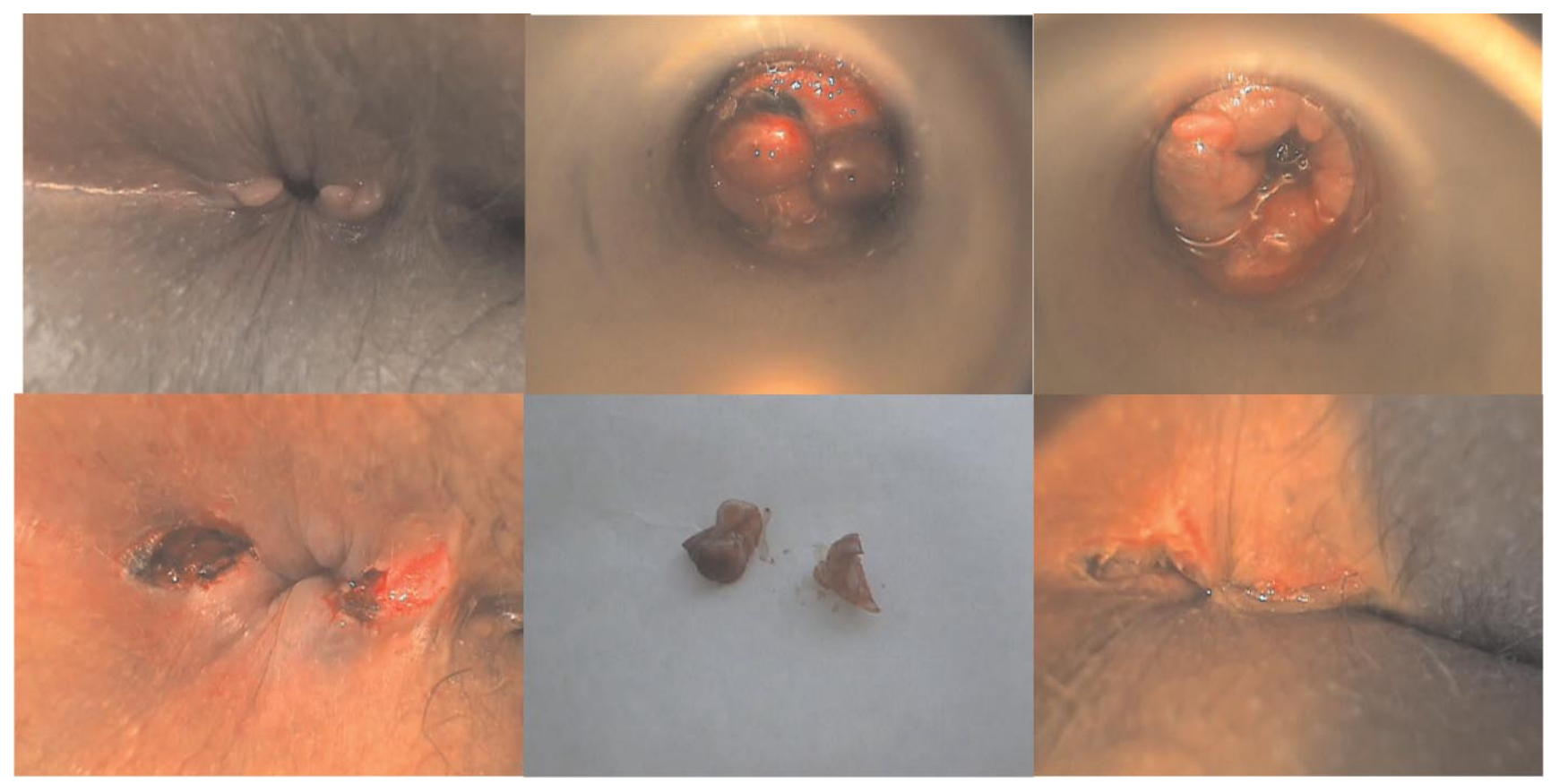

Figura 6- LE dupla e Fissurectomias (Hemoroidectomia Híbrida pós-hemorroidectomia clássica).
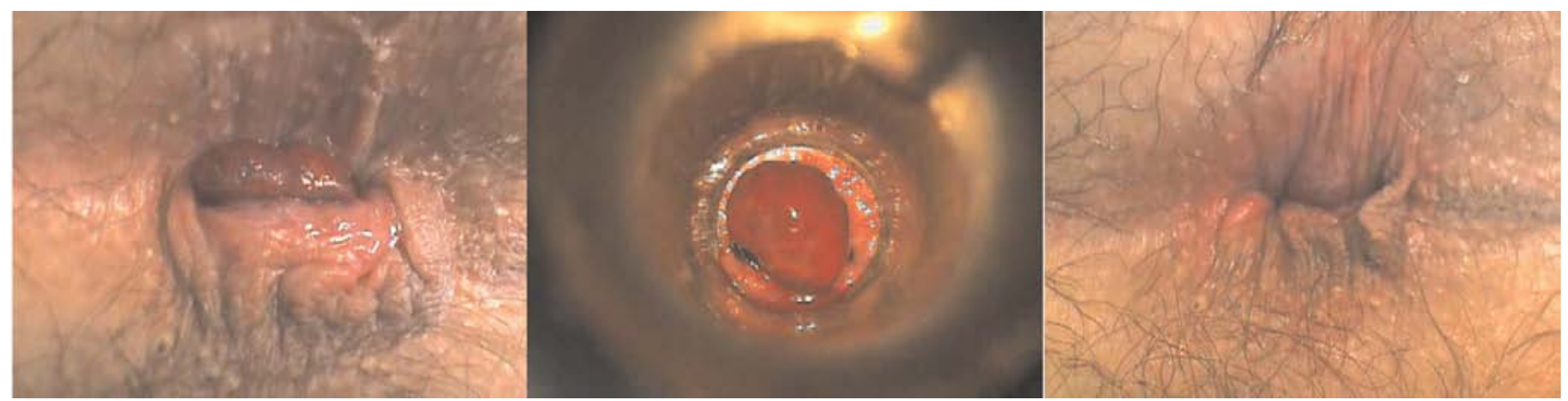

Figura 7 - Prolapso incoercível, LE e resultado imediato. 


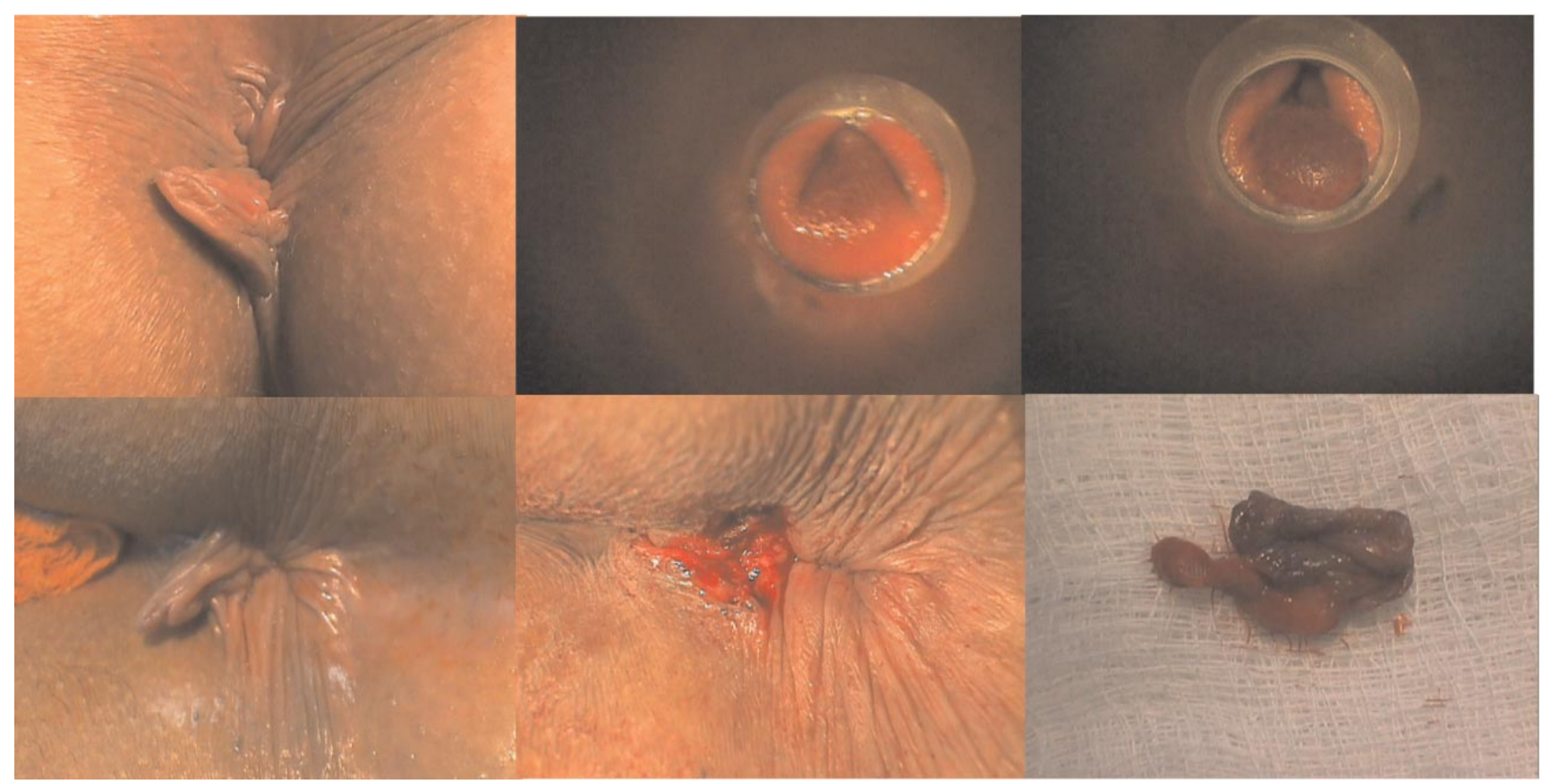

Figura 8 - LE de mamilo antero- lateral direito e Exérese de Plicoma Anterior.

\section{Tabela 1}

\section{Proctoclínica - Período de 1996 a 1999}

Submetidos à LE como tratamento exclusivo

Submetidos à Hemorroidectomia Híbrida

223

77

26

326
$68 \%$

$24 \%$

$8 \%$

$100 \%$

\section{Tabela 2}

\section{Proctoclínica - Hemorroidectomia Híbrida}

Diagnósticos

\begin{tabular}{lrr}
\hline Hemorróidas III + Plicomas & 32 & $42 \%$ \\
Hemorróidas II + Plicomas & 14 & $18 \%$ \\
Hemorróidas II + Fissuras & 7 & $9 \%$ \\
Hemorróidas III + Plicomas + Hipertonia Esf. & 5 & $6 \%$ \\
Hemorróidas II + Plicomas + Fissura Anal & 4 & $5 \%$ \\
Hemorróidas III + Plicomas + Fissura Anal & 4 & $5 \%$ \\
Hemorróidas IV + Plicomas & 3 & $4 \%$ \\
Hemorróidas III + Trombose Externa & 3 & $4 \%$ \\
Hemorróidas III + Papilites Hipertróficas & 3 & $4 \%$ \\
Hemorróidas IV + Fistuleta Sub-fissurária & 1 & $1 \%$ \\
Hemorróidas III + Pólipo Anal Fibroso & 1 & $1 \%$ \\
Total & $\mathbf{7 7}$ & $\mathbf{1 0 0 \%}$ \\
\hline
\end{tabular}




\section{Tabela 3}

\section{Proctoclínica - Hemorroidectomia Híbrida \\ História Cirúrgica Pregressa}

\begin{tabular}{lrr}
\hline Ligaduras Elásticas Prévias ( 2 a 10 anos ) & 10 & $13 \%$ \\
Hemorroidectomias Prévias & 5 & $6 \%$ \\
Hemorroidectomias ( 2 ) + Proctoplastia Prévia & 1 & $1 \%$ \\
Injeções Esclerosantes + Ligaduras Eláticas Prévias & 1 & $1 \%$ \\
\hline
\end{tabular}

\section{Tabela 4}

\section{Proctoclínica - Hemorroidectomia Híbrida \\ História Cirúrgica Pregressa}

Ligaduras Elásticas Prévias ( 2 a 10 anos )

Hemorroidectomias Prévias

Hemorroidectomias ( 2 ) + Proctoplastia Prévia

Injeções Esclerosantes + Ligaduras Eláticas Prévias

\begin{tabular}{|c|c|}
\hline 10 & $13 \%$ \\
\hline 5 & $6 \%$ \\
\hline 1 & $1 \%$ \\
\hline 1 & $1 \%$ \\
\hline
\end{tabular}

\section{Tabela 5}

\section{Proctoclínica - Hemorroidectomia Híbrida Complicações Pós-operatórias}

Plicomas Residuais

Granulomas

Espasmo Esfincteriano ( Dor Pos-operatória )

Fissuras Residuais

Hemorragia Pós-operatória

Fistuleta subcutânea

Tromboses

Polipo Fibroso pós-hematoma organizado juxta-papilar

\begin{tabular}{rr}
12 & $16 \%$ \\
5 & $6 \%$ \\
4 & $5 \%$ \\
3 & $4 \%$ \\
3 & $4 \%$ \\
2 & $3 \%$ \\
2 & $3 \%$ \\
2 & $3 \%$ \\
\hline
\end{tabular}

\section{Tabela 6}

\section{Proctoclínica - Hemorroidectomia Híbrida Tratamento das Complicações}

\begin{tabular}{lrr} 
Reintervenções em Plicomas Residuais & 12 & $16 \%$ \\
Cauterizações Químicas & 4 & $5 \%$ \\
Fissurectomias & 3 & $4 \%$ \\
Esfinterotomias Complementares & 2 & $3 \%$ \\
Hemostasias Pós-operatórias & 2 & $3 \%$ \\
Exérese de Hematoma & 1 & $1 \%$ \\
\hline
\end{tabular}

correspondidas, inclusive facultando a ampliação de suas indicações a casos mais exuberantes em que há contra-indicação da cirurgia convencional por razões clínicas imperiosas, e os resultados são totalmente satisfatórios, apesar do período de tratamento mais prolongado.

A racionalização e a associação de técnicas nos permitiu conduzir propostas e planos de tratamen- 
to bem aceitos pelos pacientes que se vêem livres da internação hospitalar e dos bloqueios raquidianos e peridurais.

Pitanga Santos afirmava que uma teoria só adquire foros de verdade quando respaldada por uma entidade de pesquisa e como dissemos anteriormente, mudar um conceito é difícil, entretanto, quando os argumentos são consistentes e os resultados convincen- tes, fica mais fácil agregarmos estes novos conceitos e preceitos.

É mister que outros serviços acolham e utilizem esta metodologia para filtrarmos experiências multicêntricas que possam detectar a real dimensão dos resultados, possibilitando a divulgação de dados que possam corroborar na escolha da abordagem ideal para o tratamento das Hemorróidas.

ABSTRACT: The goal of this work is to present a new mini-invasive approach of the Internal and External Hemorrhoids Treatment, the Hybrid Hemorrhoidectomy, that consists of an association of the Rubber Band Ligation (RBL) of the Internal Hemorrhoids with complementary withdraw of the External Piles under local anesthesia.

In a universe of 326 anal surgeries carried through in Proctoclínica in a period of 4 years, a number of 300 patients (92\%) had been submitted to mini-invasive procedures $(223(68,40 \%)$ had been submitted to RBL as exclusive treatment and $77(23,60 \%)$ to Hybrid Hemorrhoidectomy) and 26 patients $(8 \%)$ had been submitted to other surgical procedures (Hemorrhoidectomy MilliganMorgan, Fistulectomy, etc.)

The proposal approach allows minimum absenteism to the work, mini-invasivity and low pos-operative morbidity with the advantage of office and outpatient procedures, excellent tolerability and low costs.

Kew words: Hemorrhoidal Disease; Hemorrhoids; Hemorrhoidectomy; Rubber Band Ligation; Ano-Rectal Disease.

\section{REFERÊNCIAS}

1. Arullani A. et al. - Diagnosis and current treatment of Hemorrhoidal Disease Angiology 1994 jun; 45 (6pt2):560-5.

2. Barron, James - Office Ligation treatment of Hemorrhoids Dis. Col. Rectgum 1963; 6: 109-13.

3. Barwell, J. And cols - Life-threatening Retroperitonieal Sepsis after Hemorrhoid Injection Sclerotherapy - Dis. Col. Rectum 1985; 28: 188-9.

4. Bech W., Dishler W., Helms M. And Oehlert W. - Endoscopia e Biópsia do Intestino - F. K. Schattauer Verlag - 1978.

5. Bensaude, R. - Rectoscopie Sigmoïdoscopie - Libraire de L'Académie de Médecine 1956.

6. Bonelli, J. C. - Who's afraid of the dentate line? The Whitehead Hemorrhoidectomy - Amer. J. Surgery 1988; 156: 182-6.

7. Cherry D. A. and cols. - Pelvic Floor Physiology Surgical clinics of North America 1988 dec; vol 68 (6): 1217-30.

8. Cheetham, Mark J. and cols. Persistent pain and faecal urgency after Stapled Haemorrhoidectomy Lancet 2000; 356 (9231): 730-33.

9. Copé R. - Etiopathogenie de la maladie hémorroïdaire Acta Gastroent.Belgica 1994 March-April; vol LVII: 206-12.

10. Corman M. L. - Colon and Rectal Surgery J. B. Lippincott Comp. 2a Edition 1989.

11. Corman, M.L. and cols. - The New Hemorrhoidectomy Surg. Clin. North Amer. 1973 April ; 53.

12. Clay, LDIII and cols. - Early recognition and successful management of pelvic celulitis following hemorrhoidal banding Dis. Col. Rectum 1986; 29: 579-81.
13. Dennison A. R. and cols. - Hemorrhoids: nonoperative management - Surgical Clinics of North America 1988 Dec; vol 68 (60):1401-9.

14. Duhamel J. - Proctologie Salvat Editores 1974.

15. Ger R. - Surgical Anatomy of the Pelvis - Surgical Clinics of North America 1988 Dec; vol 68 (6) :1201-16.

16. Goligher, J. - Surgery of the Colon, Rectum and Anus - Salvat Editores 1979.

17. Goligher, J. And cols. - The Surgical Anatomy of the Canal Anal The Brit. Journ. Of Surgery.

18. Haas P. A. and cols. - The prevalence of Hemorrhoids Diseases of the Colon and Rectum 1983 July; vol 26 (7): 435 39.

19. Haas P. A. and cols. - The pathogenesis of Hemorrhoids Diseases of the Colon and Rectum 1984 July; vol 27 (7): 44250.

20. Hayssen, Theresa K. And cols. - Limited Hemorrhoidectomy - Results and long-term follow-up - Dis. Col.. Rectum.

21. Henry M. M. and cols. - Fecal Incontinence and Rectal Prolapse - Surgical Clinics of North America 1988 Dec; vol 68 (6): 1249-54.

22. Herold, A. And cols. - Pain after stapled haemorrhoidectomy - Lancet 2000; 356: 2187.

23. Hetzer, F.H. and cols. - Stapled vs Excision Hemorrhoidectomy: long-term results of a perspective randomized trial Arch. Surgery 2002; 137: 337-40.

24. Kaman L. And cols. - Necrotising Fascitis after injection sclerotherapy for hemorrhoids - Dis. Col. Rectum 1999; 42: 419-20. 
25. Khalil, K.H. and cols. - Randomized clinical trial of sutured versus stapled closed haemorrhoidectomy Brit. Surgical 2002; 87: 1352-57.

26. Kirsch, J.J. and cols. - Stapled vs Open Hemorrhoidectomy Crirurg. 2001; 72: 180-5.

27. Kirsner, J.B. and Shorter R.G. - Diseases of the Colon, Rectum and the Anal Canal - Williams and Wikins Edit. 1998.

28 - Khubchandani, Indru T. - Operative Hemorrhoidectomy Surg. Clin. North Amer. 1988 Dec.; 68(6): 1411-1416

29. Lileswar Kaman, L.S. and cols. - Necrotising fascitis after injection sclerotherapy for hemorrhoids Dis. Col. Rectum 199; 42: 419-20.

30. Johanson J. F. and cols. - The prevalence of Hemorrhoids and Chronic Constipation: an epidemiologic study Gastroenterology 1990; 98 : 380-86.

31. Johanson J. F. and cols. - Optimal non surgical treatment of Hemorrhoids: a comparative analysis of Infrared Coagulation, Rubber Band Ligation and Injection Sclerotherapy Am J. Gastroenterology 1992 Nov ; 87 (11): 1600-6

32. Johanson J. F. - Constipation is not a risk factor of Hemorrhoids: a case-control study of potential etiological agents The Am. J. of Gastroenterology 1994; Vol 89(11) : 1981-86.

33. Lloyd Davis and cols - Modified Longo's Hemorrhoidectomy - Dis. Col. Rectum 2002 March; 45(3): 416-7.

34. Loder P. B. et al. - Haemorrhoids: pathology, pathophisiology and aetiology Br. J. surgery 19941994 July; 81 (7): 946-54.

35. Magriná, Emilio de los Rios - Atlas de Coloproctologia Salvat Ed. - 1978.

36. Maw, Andrew and cols - Retroperitoneal sepsis complicating stapled hemorrhoidectomy - Dis. Col. Rectum 2002 jun; 45(6): 826-28.

37. Marshak R.H., Lindner A.E. and Maklansky - Radiology of the Colon - W.B. Saunders Co. 1980.

38. Mazier, W Patrick - Hemorrhoids, Fissures and Pruritus Dis. Col. Rectum 1994 Dec; 74 (6): 1277-92.

39. Mehigan, B.J. and cols - Stapling procedure for hemorrhoids versus Milligan-Morgan Haemorrhoidectomy: randomized controlled trial - Lancet 2000; 355: 782-5.

40. Modesto V. L. - Sexually transmitted diseases and anal manifestations of Aids Surgical Clinics of North America 1994 Dec; 74 (6): 1433-64.

41 - Molloy, R.G. and cols. - Life-threatening pelvic sepsis after stapled haemorrhoidecftomy - Lancet 2000; 355(9206): 810

42. Murphy, K.J. - Tetanus after Rubber Band Ligation Haemorrhoidectomy - Brit. Med. Journal 1978; 1: 1590-1.

43. Ohara, v.s. - Fatal Clostridial infection following Hemorrhoidal Banding - Dis. Col. Rectum 1985; 28:188-9.

44. O'Regan, P.J. and cols. - Disposable device and a minimally invasive technique for Rubber Band Ligation of Hemorrhoids - Dis. Col. Rectum 1999 May; 42(5): 683-5.
45. Oliver G. C. and cols. - Neoplasms of the Anus - Surgical Clinics of North America 1988 Dec ; Vol 68 (6): 1475-90.

46. Pernici, Luigi M and cols. - Early and late (ten years) experience with Circular stapler Hemorrhoidectomy - Dis. Col. Rectum 2001 Jun; 44(6): 836-41.

47. Pescatori M. and cols - Diagnostic Anorectal Functional Studies - Surgical Clinics of North America 1988 Dec; Vol 68 (6): 1231-48.

48. Quevedo, Bonilli - Septic complication of Hemorrhoidal Banding - Arch. Surg. 1988; 123: 650-1.

49. Ribbans, W.J. - Retroperitoneal abscess following Sclerotherapy for Hemorrhoids - Dis. Col. Rectum 1985; 28 : 188-9.

50. Ripeti, V. and cols. - Rectal perforation, retroperitonium and pneumo-mediastinum after Stapling Procedure for Prolapsed Hemorrhoids Medline.

51. Ross, P. - Haemorrhoid Surgery revised - Lancet 2000; 355 : 1648.

52. Russell, T. R. and cols. - Hemorrhoidal Banding: a warning. Dis. Col. Rectum 1985; 28: 291-3.

53. Santos R. P. - Etiopatogenia das Hemorróidas - Rev Bras Colo-Proct 1993; 13(2): 55-66.

54. Scarpa, F.J. and cols - Pelvic cellulitis - a life-threatening complication of Hemorrhoidal Banding surgery Surgery 1988; 103: 383-5.

55. Siddharth P. and cols. - Colorectal Neurovasculature and Anal Sphincter Surg Clinics of North America 1988 Dec; Vol 68 (6): 1185-200.

56. Singer, Marc A. And cols. - Early experience with Stapled Hemorrhoidectomy in the United States Dis. Col. Rectum 2002; 45: 360-7.

57. Shemsh, C.I. - Sever Complication of Rubber Band Ligation of Internal Hemorrhoids - Dis. Col. Rectum 1987; 30: 199200.

58. Sun W. M. et al. - Haemorrhoids are associated not with hypertrophy of the internal sphincter, but with hypertension of the anal cushion Br. J. Surgery 1992 Jun;79(6):592-4.

59. Thomson W. H. F. - The nature of Hemorrhoids Br. J. Surgery 1975; vol 62 : 542-52.

60. Todd, Yan P. - Colon Rectum and Anus - Gen. Ed.:Rob, C.

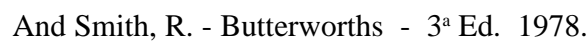

61. Wolf, B.G. and cols. - The Whitehead Hemorrhoidectomy: an unjustly maligned procedure - Dis. Col. Rectum 1998; 31 : 587-90.

62. Yik-Hong Ho and cols. - Stapled Hemorrhoidectomy - Cost and effectiveness. Randomized, controlled trial including Incontinence scoring, Ano-rectal Manometry and Endo-anal Ultrasound Assessments at up to three months. - Dis. Col. Rectum 2000; 43: 1666-75.

63. Wai-lun Law and cols. - Triple Rubber Band Ligation for Hemorrhoids - Prospective, randomized trial of use of local anesthetic injection - Dis. Col. Rectum 1999 March; 42(30: 363-6. 
64. Welti, Henry - Chirurgie du Colon - Libraire de L'Académie de Médecine 1960.

65 Wither, D.G. and cols. - An unusual complication of Rubber Banding Ligation of Hemorrhoids - Dis. Col. Rectum 1987; 30: $137-40$.

66. Excisional Hemorrhoidectomy: A Distant Memory? 17th International Colorectal Disease Symposium - The Colorectal Department at Cleveland Clinic Florida - 2006
67. Longo, A. - Treatment of hemorrhoids disease by reduction of mucosa and hemorrhoidal prolapse with a circular suturing device: a new procedure. - Sixth world Congress of Endoscopic surgery - Rome - Italy - Monduzzi Publishing co. - 1998 : 777-84.

Endereço para correspondência: HAROLDO ALFREDO SANTOS

Rua Caldeira Brant, 201

31030-180 - Belo Horizonte - MG

E-mail: haroldo.bh@terra.com.br 